begreifen, dass jetzt dieses nationale Werk in Deutschland losgeht [...]. Sie sollten jetzt endgültig über Ihren Schatten springen“ (CDU 30.06.2011: 13368).

In der Erzählung von nationaler Gemeinschaft und Kultur sowie im Appell an Einigkeit zeigt sich deutlich die pädagogische Dimension des nationalen Narrativs: Die Historizität der Nation wird behauptet, aber ihre Ambivalenz gleichzeitig verdrängt die Konfliktivität in der Geschichtlichkeit von „Energiewende“, die sich im Zusammenhang mit den Protesten gegen Atomkraft und für erneuerbare Energien seit den 70er Jahren und nach „Fukushima“ sowie gegen die Laufzeitverlängerung 2010 zeigt, wird durch die Berufung auf eine nationale Einheit verdeckt. So erscheint die „Energiewende“ im Bundestag letztlich als enthistorisierte „Energiewende“.

Im Zuge der nationalen und neokolonialen Externalisierungsprozesse, die sich über den „Energiewende“-Diskurs hinweg entfalten, lassen sich wie auch anhand der zuvor analysierten Dichotomien die hegemoniale Gesellschafts- und Naturverhältnisse nachzeichnen. Die Auslagerung von Sozialem und Ökologischem aus dem unter rein ökonomischen Kriterien betrachteten Politischen wird durch die Konstruktion nationaler Identität weiter verstärkt und in ihrer neokolonialen Dimension vertieft. Insgesamt wurde deutlich, dass in Bezug auf die aufgezeigten Dichotomien in der hegemonialen Diskursformation keine aussichtsreichen Ansätze für Vermittlung bspw. im Sinne der Kategorie der (Re)Produktivität aufgezeigt werden.

\title{
6.5 POTENZIALE FÜR EINE HERRSCHAFTSÄRMERE ZUKUNFTSGESTALTUNG IM DISKURS UM ,ENERGIEWENDE“
}

In Kapitel 3.2.2 wurde ,Potenziale für eine herrschaftsärmere Zukunftsgestaltung ‘ als weiteres Kriterium Vorsorgenden Wirtschaftens eingeführt. Es fragt in dieser Arbeit vor allem nach den Potenzialen, die sich im energiepolitischen Diskurs im Bundestag für eine herrschaftsärmere Zukunftsgestaltung zeigen, wozu es in Hinblick auf den Umgang mit Externalisierungen bereits im vorausgehenden Kapitel erste Rückschlüsse gab. Dies wird nun mit der Frage nach Gerechtigkeit ${ }^{64}$ - als Legitimation von politischen Praktiken, die auf eine herrschaftsärmere Zukunftsgestaltung zielen im Kontext des Ansatzes von ,Affirmation“ und ,Transformation' nach Fraser (2001: 47ff.) sowie der Frage nach Eigentum (Biesecker/von Winterfeld 2011) verbunden.

In Kapitel 5.3.1 wurden die politischen Praktiken, die sich im Kontext des fantasmatischen Narrativs, ökologische Modernisierung' zeigen, als reformistisch ausgerichtet klassifiziert, jedoch wurde wegen einer strukturell externen Verortung der Hindernisse/Gefahren auf eine beharrende Wirkung bezüglich ökonomischer Strukturen verwiesen. Im Rahmen der in Kapitel 5.3.2 erfolgten Klassifizierung wurden politischen Praktiken im Kontext des Narrativs ,marktwirtschaftliche Energiepolitik sowohl in Bezug auf die externe Gefahrenverortung als auch auf die am Status-Quo orientierte Glücksverheißung eine beharrende Ausrichtung attestiert und reformisti-

64 Für den begrifflichen Umgang mit ,Gerechtigkeit` in der vorliegenden Arbeit vgl. Fußnote 43 in Kapitel 3. 
sche Ansätze nur insofern erkannt, als dass auf Veränderung ausgerichtete Praktiken insbesondere aufgrund der ,neuen Lage“ nach „Fukushima“ angeeignet werden. Eine gemeinsame Betrachtung politischer Praktiken im Rahmen der beiden Narrative in Bezug auf ihre Wirkung im Spannungsfeld zwischen Transformation, Reform und Beharrung macht insofern Sinn, als dass sie, wie in Kapitel 5.4 gezeigt, spätestens in der zweiten Hälfte der 17. Legislaturperiode im gemeinsamen Narrativ eines ,technologisch-managementorientierten Ökokonsenses` aufgehen. Da weder zuvor noch danach das parallel verlaufende fantasmatische Narrativ ,sozial-ökologischer Umbau' aufgrund seiner marginalisierten Diskursposition die Ausrichtung politischer Praktiken entscheidend beeinflusst, bleibt es in der folgenden Analyse über den emanzipatorischen Gehalt dieser politischen Praktiken weitestgehend außer Betracht.

Sowohl das Narrativ ,ökologische Modernisierung' als auch das Narrativ ,markwirtschaftliche Energiepolitik' setzen an einem affirmativen - im Gegensatz zu einem transformativen - Umgang mit Ungerechtigkeit an, was sich in Bezug auf Anerkennung zum Beispiel an einem fehlenden Entgegenwirken gegen Externalisierung zeigt (vgl. Kapitel 6.4). Politische Praktiken, die sich im Kontext der Narrative zeigen, zielen nicht auf die Veränderung der Ursachen sozial-ökologischer Krisenphänomene in den kapitalistischen Produktionsverhältnissen, sondern auf eine Abschwächung der ökologischen Folgen dieser Verhältnisse vor allem durch technologische Verbesserungen im Produktionsprozess, auch wenn zumindest anfangs innerhalb des Narrativs ,ökologische Modernisierung ' interne Hindernisse in den Produktionsverhältnissen thematisiert werden. Der herrschaftlichen Strukturierung gesellschaftlicher Verhältnisse sowie gesellschaftlicher Naturverhältnisse mitsamt der zugrundeliegenden Externalisierungsstrukturen, die mit der letztlich marktökonomischen Ausrichtung politischer Praktiken verbunden ist, wird aber nicht entgegengewirkt. Exemplarisch werden nun für den Energiebereich einige Instrumente affirmativer Gerechtigkeitspolitiken analysiert, um zu überprüfen, inwiefern sie sich als geeignet erweisen, Herrschaft und Ungerechtigkeit abzubauen, bzw. inwiefern sie Ungerechtigkeiten sogar noch verstärken. Politische Praktiken, die auf Transformation ausgerichtet sind, ließen sich im Rahmen der Diskursanalyse außerhalb einer marginalisierten Position nicht ausmachen, weswegen die Bewertung bezüglich transformativer Umverteilung oder Anerkennung hier zwangsläufig in den Hintergrund rückt.

\section{Ökosteuer}

Ein Beispiel für affirmative Gerechtigkeitspolitik im Kontext des Narrativs ,ökologischer Modernisierung ' ist die Ökosteuer. Sie birgt zunächst das Potenzial, affirmative Umverteilung mit transformativer Anerkennung zu verbinden, da sie an der Überwindung von Externalisierungen ansetzt: Externe Kosten durch die ökologischen Auswirkungen des (Energie)Produktionsprozesses sollen internalisiert werden. Gleichzeitig können so umweltschädliche externe Effekte des Produktionsprozesses monetär und somit ökonomisch sichtbar gemacht werden, was im Idealfall zu einer höheren Anerkennung von Naturleistungen führen kann. Allerdings wird die Einführung der Ökosteuer im Bundestag nicht nur mit der Internalisierung externer Effekte („Naturverbrauch“; SPD 03.03.1999: 1835f.) gerechtfertigt, sondern in erster Linie mit der Senkung der Sozialversicherungsbeiträge, für die die Einnahmen aus der 
Ökosteuer verwendet werden. ${ }^{65}$ Naturleistungen kommen so in den Debatten eher selten in den Blick, der Anerkennungseffekt wird konterkariert. Eine Vermittlung zwischen Arbeits- und Naturproduktivität im Sinne von (Re)Produktivität findet so gerade nicht statt. Stattdessen werden die Preise der ,Faktoren“ Naturverbrauch“ und „Arbeit“ (SPD: 03.03.1999: 1835f.) im Rahmen einer marktwirtschaftlichen KostenNutzen-Rechnung optimiert. Hier zeigt sich nach Biesecker/von Winterfeld (2014: 4), dass die Externalisierung „nicht mittels Internalisierung aufgelöst werden“ kann, weil das ökonomische und politische System nicht ohne die Ausbeutung des Wertlosen - in dem Fall der Natur - funktioniere. Da die Einnahmen aus der Ökosteuer kaum für umweltpolitische Zwecke eingesetzt werden, wird ein möglicher sozialökologischer Umverteilungseffekt unterlaufen. Die PDS kritisiert zudem eine ,soziale[...] Schieflage“ (PDS 25.11.1999: 6748): Während private Haushalte mit geringem Einkommen prozentual am höchsten belastet werden, sollen Unternehmen, die durch ihre Produktionsweise ökologische Schäden mit verursachen, durch die Steuer sogar entlastet werden. Energieintensive Unternehmen werden überwiegend von der Ökosteuer befreit und auch kleinere Unternehmen sollen im Saldo durch die Senkung der Lohnnebenkosten von der Steuer profitieren. ${ }^{66}$ Ökologische Auswirkungen spielen in den Argumentationen zur Ökosteuer kaum eine Rolle. Der Versuch, aktiv mit Externalisierung umzugehen, wird auf halbem Wege abgebrochen, weswegen ihre Wirkung auf Anerkennungsebene affirmativ bleibt. Auch bezüglich der Umverteilung bleibt die Wirkung, wie beschrieben, affirmativ, wenn überhaupt von einer Umverteilung im Sinne der Verschiebung von Eigentum von oben nach unten gesprochen werden kann. Vielmehr wird der Ökosteuer ein umgekehrter Effekt attestiert. ${ }^{6768}$

65 Vgl. z.B.: „Als vor über 10 Jahren über Ökosteuern diskutiert wurde, war die verbreitete Meinung, man brauche das ganze Geld für die ökologischen Wohltaten. So ähnlich hat es auch Frau Merkel heute Nachmittag gesagt. Das ist aber ein Denkfehler. Damit bekommt man nicht das notwendige Paket, auf Grund dessen sich auch die Arbeitnehmerseite voll mit diesem Reformprojekt identifizieren kann. Es ist, wie Herr Minister Lafontaine richtig ausgeführt hat, in einer Situation eines Überschusses von Arbeit, einer hohen Arbeitslosigkeit, und eines eigentlich knappen Gutes Natur über alle Parteigrenzen hinweg erforderlich, die Kosten des Faktors Arbeit zu senken und die Kosten des Faktors Naturverbrauch zu erhöhen“ (SPD 03.03.1999: 1835f.). Vgl. z.B.: „Die Mittel der Ökosteuer werden zur Senkung des Rentenbeitrags verwandt, der im Jahre 2000 von jetzt 19,5 Prozent auf 19,3 Prozent sinkt [...].Das ist durchaus einen Beifall wert, denn wir losen damit ein weiteres Wahlversprechen ein: die Senkung der Lohnnebenkosten“ (SPD 25.11.1999: 6670). Vgl. z.B.: „Wir folgen einer Logik, indem wir eine der Hauptbelastungen der Unternehmen durch eine sinnvolle Maßnahme, nämlich die ökologische Steuerreform, verringern“ (SPD 03.03.1999: 1838). Vgl. z.B.: „Ja, er hat gesagt: Endlich wurde die Arbeitskraft verbilligt, endlich wurde etwas getan, um die Ressourcen zu besteuern. Damit werde eine Wende eingeleitet“" (GRÜNE 25.11.1999: 6747).

66 So bezeichnen die GRÜNEN die Ökosteuer als „das größte Entlastungsprogramm, das es bei den Lohnnebenkosten gibt" (GRÜNE 09.09.2004: 11253).

67 Die PDS kritisiert: „Abgesehen von der sozialen Schieflage der rotgrünen Konstruktion, ist das Paket in umwelt- wie haushaltspolitischer Hinsicht eine Katastrophe. Die Ökosteuer schafft keine zusätzliche Finanzierungsgrundlage für den ökologischen Umbau, weil deren 


\section{Erneuerbare Energien Gesetz (EEG)}

Ähnlich wie die Ökosteuer bietet auch die Einführung des EEG das Potenzial, auf Anerkennungsebene transformative Wirkung zu entfalten, in Verbindung mit einer affirmativen Umverteilungspolitik. Denn zum einen gerät durch die erneuerbaren Energien (Sonne, Wind, Wasser) Naturproduktivität in den Blick, zum anderen werden Produktionsmittel durch den Einspeisevorrang und die feste Einspeisevergütung von wenigen großen Unternehmen, die überwiegend konventionelle Energie produzieren, zu vielen kleinen Produzent*innen erneuerbarer Energien umverteilt. Dadurch werden zahlreiche kleine Akteur*innen in den Produktionsprozess einbezogen, Herrschafts-Eigentum in Form von monopolisierter Energieproduktion wird zurückgedrängt. Bei einer konsequenten Umsetzung einer solchen Umverteilungspolitik unter Aufbrechen der Dichotomie zentral/dezentral im Zuge einer grundliegenden Umstrukturierung des Energiesektors, würde das EEG auf Umverteilungsebene sogar transformative Wirkung entfalten. Zudem werden die Rechte zukünftiger Generationen anerkennend einbezogen, was den transformativen Charakter auf Anerkennungsebene durch Zugrundelegung eines intergenerationellen Gerechtigkeitsverständnisses stärkt: Die Förderung erneuerbarer Energien diene auch „dazu, die Subvention des herkömmlichen Energieverbrauchs mit seinen Umweltschäden durch die nächsten Generationen, die diese Umweltschäden ,abbezahlen " müssen“ zu beenden (SPD 25.02.2000: 8438f.). Bezüglich der Externalisierung von Umweltschäden ist also zunächst in der Formulierung der politischen Praktiken ein Entgegenwirken erkennbar. Allerdings zeigen sich solche Artikulationen in den Debatten um das EEG eher selten, es wird dagegen im Kontext des Narrativs ,marktwirtschaftliche Energiepolitik vor allem mit den marktökonomischen Potenzialen argumentiert (vgl. z.B. Kapitel 5.2.3), was auch im Rahmen des Narrativs ,ökologische Modernisierung ‘ eine Rolle spielt. Die Förderung erneuerbarer Energien wird als „Schlüsselfaktor für die Entwicklung gerade der deutschen Wirtschaft“ (SPD 27.06.2002: 24776) dargestellt. So wird nicht nur die Chance vertan gesellschaftliche Naturverhältnisse, oder gar deren herrschaftliche Strukturierung, zu adressieren, sondern auch die Veränderung der Energieproduktionsstrukturen durch erneuerbare Energien stärker hervorzuheben. Die somit zunächst noch als affirmativ zu kennzeichnenden Umverteilungsprozesse im Rahmen des EEG werden allerdings spätestens mit den Novellen 2014 und 2016/2017 weiter abgeschwächt, worauf ich an späterer Stelle noch näher eingehe.

Einnahmen aus den Energiesteuern fast vollständig für die Senkung der Lohnnebenkosten verwendet werden“ (PDS 25.11.1999: 6748).

68 Dies bestätigt sich auch in zwei Studien des DIW, die dieses Anfang 2019 als Bilanz zum 20 jährigen Bestehen der Ökosteuer veröffentlicht: „Die vor 20 Jahren in Kraft getretene ökologische Steuerreform hat ihre Ziele - die Entlastung der Rentenversicherung und eine bessere Energieeffizienz - nur zum Teil erreicht. Sie generiert Steuereinnahmen, die bis heute das Rentensystem entlasten. Sie hat allerdings nicht wie gewünscht dazu geführt, dass der Energieverbrauch und somit die Treibhausgasemissionen in Deutschland nachhaltig sinken. [...] Weil die Ausgaben für Energie in ihrem Budget schwerer wiegen und sie kaum von Beitragssenkung und Rentenerhöhung profitieren, sind einkommensschwache Haushalte relativ von der Ökosteuer mehr betroffen als Haushalte mit mittleren oder hohen Einkommen“ (www.diw.de - Ökosteuer). 
Auch auf Anerkennungsebene bleibt die Wirkung des EEG folglich eine affirmative, da spätestens im Rahmen des ,technologisch-managementorientierten Ökokonsenses ‘ Artikulationen, die unter dem Narrativ der ,ökologischen Modernisierung' noch auf das Aufbrechen etwa der Dichotomie zentral/dezentral oder durch Einbeziehen der Naturproduktiviät der Dichotomie produktiv/reproduktiv abzielten, im Diskurs ausbleiben. Daran zeigt sich, dass affirmative politische Praktiken, z.B. im Rahmen affirmativer Umverteilungsmaßnahmen, weniger zum Aufbrechen von Dichotomien auf Anerkennungsebene geeignet sind und diesbezüglich eine eher beharrende Wirkung entfalten (vgl. auch Fraser 2001: 52f.). Dies wird unter Verweis auf das ,Umverteilungs-Anerkennungs-Dilemmas‘ nach Fraser (2001: 27) später ebenfalls anhand des Beispiels der EEG-Novellen von 2014 und 2016/2017 weiter verdeutlicht.

\section{Emissionshandel}

Auch die Einführung des Emissionshandels als Instrument der EU-Klimapolitik in der 15. Legislaturperiode (und seine Anpassung in der 16. und 17. Legislaturperiode) ist ein Beispiel dafür, dass eine affirmative Umverteilungspolitik auf Anerkennungsebene keine transformative Wirkung entfaltet, auch wenn diese in der ursprünglichen Intention artikuliert wird. Diese zielt ähnlich wie bei der Ökosteuer auf die Internalisierung externer Effekte $\mathrm{ab}$ - in diesem Fall durch eine Bepreisung von $\mathrm{CO}_{2}$ - und somit auf die Einbeziehung von Natur in ökonomische Prozesse. Der Emissionshandel hat das Potenzial, Naturleistungen (z.B. den Abbau von $\mathrm{CO}_{2}$ ) sichtbar zu machen und dadurch deren Anerkennung zu steigern sowie gleichzeitig die Einnahmen aus dem Verkauf der Zertifikate für die Erneuerung bzw. Wiederherstellung von Natur zu verwenden. Somit wird potenziell nicht nur die Klimaschädlichkeit von Produktionsweisen sichtbar, sondern gleichzeitig können Gewinne aus klimaschädlicher Produktion abgeschöpft und umverteilt werden, wobei neben dem Umverteilungsaspekt auch der Anreiz zur $\mathrm{CO}_{2}$-neutralen Produktion im Vordergrund steht. Allerdings zeigen sich in der Konstruktion und Wirkung des Emissionshandel zahlreiche Aspekte, die sowohl den transformativen Potenzialen im Hinblick auf Anerkennung als auch den Potenzialen für affirmative Umverteilung zuwider laufen. So ist zunächst die Schaffung von Privateigentum an $\mathrm{CO}_{2}$ für den Handel am Markt notwendig. Es zeigt sich auch hier, dass hierarchisch strukturierte gesellschaftliche Naturverhältnisse nicht durch Internalisierung des Externen in Form eine Bepreisung überwunden werden können. Stattdessen wird Gemeinschaftsgut privatisiert und private Eigentumsrechte, Herrschafts-Eigentum, werden ausgedehnt (Biesecker/von Winterfeld 2011: 137). Dieses Privateigentum wird dann in Form von Luftverschmutzungsrechten an Unternehmen ausgegeben. Die Einbindung der Luftverschmutzungsrechte erfolgt im Zuge marktorientierter Artikulationsmuster (,gemeinsam noch mehr auf den Markt setzen“ (SPD 28.05.2004: 10248f.)) und soll gemäß dieses marktwirtschaftlichen Verständnisses zu einer optimalen Verteilung des Eigentums führen. ${ }^{69}$ Diese Marktorientierung des Emissionshandels bildet sich in den entsprechenden StoryLines (vgl. Kapitel 5.2.3 und 5.2.4) nicht nur in den Artikulationen von rot-grün ab,

69 Vgl. z.B.: „Veraltete Anlagen müssen dann zukaufen und neue Anlagen können, weil sie effizienter sind, Zertifikate am Markt verkaufen und haben so einen Vorteil im Emissionshandel“" (SPD 28.05.2004: 10249). 
sondern auch von CDU/CSU und FDP. ${ }^{70}$ Jedoch werden die Zertifikate in der ersten Phase zu großen Teilen nicht verkauft, sondern an die Unternehmen auf Grundlage von im Vorhinein und zu Beginn großzügig kalkulierten erwarteten Emissionen verschenkt. ${ }^{71}$ Die LINKE kritisiert den Effekt auf der Umverteilungsebene: „Die Sache ist doch die, dass die Kraftwerksbetreiber Milliarden an leistungslosen Extraprofiten einfahren, weil ihnen 91 Prozent der Emissionsrechte ${ }^{72}$ geschenkt werden, sie aber den Handelspreis der Zertifikate auf den Strompreis umlegen“ (LINKE 02.07.2009: 25881). Durch die emissionshandelsbedingte Erhöhung der Strompreise verdienen auch Anlagenbetreiber, die nicht emissionshandelspflichtig sind, wie Betreiber von Atomkraftwerken (vgl. auch Brand u.a. 2013: 2). Die LINKE rechnet mit ca. 18 Milliarden Euro an Extragewinnen bis 2012. ${ }^{7374}$ „Das ist ein Skandal, angesichts klam-

70 Vgl. z.B.: Es gehe „um nicht weniger als die Umstellung in der Umweltpolitik von der bisherigen reinen Ordnungspolitik hin zu einem marktwirtschaftlichen Instrument" (FDP 28.05.2004: 10239). Vgl. z.B.: „Meine sehr geehrten Damen und Herren, bei der Umsetzung des Emissionshandels hat die Union besonderen Wert darauf gelegt, dass der Aspekt, dass vorsorgender Umweltschutz mit Wirtschaftswachstum und Arbeitsplatzsicherung vereinbar ist, in den Vordergrund gestellt wird. sind, dass es gelungen ist, im TreibhausgasEmissions-handelsgesetz einen Passus zu verankern, der für neu zu gründende Unternehmen ebenso wie für Betriebserweiterungen hinreichend Emissionsberechtigungen vorsieht“ (CDU 28.05.2004: 10236).

71 Die Anzahl der ausgegebenen Zertifikate soll dann jährlich reduziert werden. Bemessen wird die Anzahl der ausgegebenen Zertifikate am sogenannten ,Cap', einer festgelegten Emissionsobergrenze, die Emissionen zu einem knappen Gut machen soll. 2019 beträgt der Lineare Reduktionsfaktor der ausgegebenen Zertifikate (,Cap-Absenkung“) jährlich 1,74\% (BMU 2018: 2).

72 In der ersten Phase von 2005-2007 wurden beinahe 100\% der Zertifikate kostenlos ausgegeben. Das Zitat bezieht sich auf die zweite Phase (2008-2012), in der ca. 9\% der Zertifikate versteigert wurden (www.umweltbundesamt.de - Emissionshandel).

73 Vgl. z.B.: „So kommt eine im Juni 2008 vorgelegte Studie des Öko-Instituts im Auftrag des WWF Deutschland zu dem Ergebnis, dass diese Extragewinne rund 35,5 Milliarden Euro, also rund 7 Milliarden Euro pro Jahr betragen. Dabei wurde ein $\mathrm{CO}_{2}$ Zertifikatepreis von 25 Euro angesetzt. Nun liegt momentan der Preis aufgrund der tiefen Wirtschaftskrise nur bei 13 Euro. Das kann sich schnell ändern, aber selbst wenn wir mit diesem Wert rechnen, kommen wir in einem Überschlag auf wenigstens 18 Milliarden Euro Extraprofite bis 2012. Ich meine, das sind ganz erkleckliche Sümmchen. Mir würde eine Menge einfallen, was man damit bezahlen könnte. Die Bundesregierung hat es jedoch bislang strikt abgelehnt, in irgendeiner Form die Windfall Profits zu besteuern. Es gibt bislang keine Hinweise darauf, dass die Bundesregierung die in anderen Ländern diskutierte Besteuerung dieser leistungslosen Zusatzgewinne wenigstens einmal thematisiert oder rechtlich geprüft hat" (LINKE 02.07.2009: 25881f.).

74 Eine 2011 vom Öko-Institut im Auftrag des WWF durchgeführte Studie (Öko-Institut 2011) bestätigt diese Einschätzung nachträglich: „Da der $\mathrm{CO}_{2}$-Preis im Bereich der Stromerzeugung auf den Strompreis umgelegt wird, konnten die Stromerzeuger im Zeitraum von 2005 bis 2012 umfangreiche Zusatzerträge realisieren. Dies zeigt eine aktuelle Untersuchung des Öko-Instituts im Auftrag des WWF Deutschland. Insgesamt haben die Stromer- 
mer Kassen und Hartz IV!“ (LINKE 02.07.2009: 25881f.). So wirke der Emissionshandel als „Gelddruckmaschine für Energiekonzerne“ (Brouns/Witt 2008 zit. in Biesecker/von Winterfeld 2011: 137). Durch die Unterwerfung unter die Nutzenmaximierungs-Rationalität ist zudem auf Anerkennungsebene nicht von einem Entgegenwirken zur Kultur/Natur Dichotomie auszugehen: „Vielmehr wird durch diese Form der Schaffung von Herrschafts-Eigentum die Ökonomisierung der Natur weitergetrieben“ (Biesecker/von Winterfeld 2011: 140). Brand u.a. (2013a: 2) schließen für den Emissionshandel:

„Durch den Emissionshandel wird Klimaschutz abhängig von Marktbewegungen und auch von Marktmacht, hinter denen nicht primär das Interesse steht, das Klima zu schützen. Vielmehr ist es umgekehrt: Die Marktbewegungen sind Resultat von Interessen, die den ,Klimaschutz a als Mittel nutzen, um Geld zu machen, ohne zum Klimaschutz beizutragen“ (Brand u.a. 2013a: 2).

Durch die leistungslosen Extraprofite (sog. ,windfall profits“ (Helbig 2010)) wird die angestrebte affirmative Umverteilung in ihr Gegenteil verkehrt. ${ }^{7576}$ Auf Anerkennungsebene wird zudem die bestehende hierarchische Strukturierung gesellschaftlicher Naturverhältnisse reproduziert, da das zunächst durch Bepreisung zu internalisieren intendierte Naturprodukt - hier in Form von Rechten an $\mathrm{CO}_{2}$-Emissionen - in Unterordnung zur industriellen Energieproduktion kostenlos angeeignet wird.

\section{Atomausstieg und Laufzeitverlängerung}

Aspekte affirmativer Gerechtigkeitspolitik zeigen sich auch im Atomausstiegsbeschluss der 14. Legislaturperiode: Ohne die Energieversorgungsstrukturen grundle-

zeuger so zwischen 35,6 und 38 Milliarden Euro Mehreinnahmen verbuchen können. Die Unternehmen der energieintensiven Industrie erzielten Erträge von rund 840 Millionen Euro" (www.oeko.de - Zusatzgewinne).

75 Es deutet sich nun allerdings eine Veränderung im Emissionshandel an, die den negativen Auswirkungen entgegenwirken soll. Ob dadurch Potenziale für den Klimaschutz erreicht werden, wird sich zeigen. So tritt Anfang 2019 eine Reform des Emissionshandels in Kraft, die bereits 2018 zu starken Preiserhöhungen der Emissionszertifikate führt. Die zuvor sehr niedrigen Preise boten kaum einen Anreiz für die Einsparung von Emissionen. Der Zertifikate-Überschuss soll nun schneller als bisher abgebaut werden. „Mit dieser Reform soll der Emissionshandel ab 2020 endlich Wirkung entfalten für den Klimaschutz. Denn bisher war der Emissionshandel aufgrund eines Überangebotes an Zertifikaten kein wirksames Klimaschutzinstrument [...]“(Agentur für Erneuerbare Energien b 2018). Zwar wird bereits seit 2013 ein Großteil der ausgegebenen Zertifikate versteigert und nicht mehr verschenkt, allerdings werden Zertifikate an Unternehmen, die durch „Abwanderung von Industrieproduktion oder vorgelagerten Anlageinvestitionen in das nicht vom Emissionshandel regulierte Ausland (sogenanntes Carbon Leakage)“ (www.bmwi.de-Emissionshandel) betroffen sein könnten, weiterhin kostenfrei ausgegeben (BMU 2018: 5).

76 Auf die Beförderung neokolonialer Strukturen durch den seit der zweiten Phase des Emissionshandels (2008) möglichen außereuropäischen Ausgleich von Emissionen in sogenannten Entwicklungsländern im Rahmen des Clean Development Mechanismus CDM gehe ich an dieser Stelle nicht ein (vgl. dazu Dietz/Brunnengräber 2008). 
gend zu ändern werden die Rechte zukünftiger Generationen an den „natürlichen Lebensgrundlagen“ (Koalitionsvertrag 1998: 1) anerkannt und in die politische Entscheidungsfindung einbezogen. ${ }^{77}$ Mit dem Beschluss zur Laufzeitverlängerung der 17. Legislaturperiode wird dieses Entgegenwirken gegen die Externalisierung von Natur sowie von zukünftigen Generationen jedoch konterkariert: Die Rechte zukünftiger Generationen an den natürlichen Lebensgrundlagen in Bezug auf die Folgen der Atomenergie werden ausgeblendet, ebenso wie die Naturleistungen, die für die Bereitstellung, den Abbau und die Entsorgung hochradioaktiver Ressourcen notwendig sind. Auf Anerkennungsebene werden somit bestehende Externalisierungen verstärkt, wobei Natur weiterhin aus den ökonomischen Prozessen ausgelagert und abgewertet wird. Auf Umverteilungsebene kann im Kontext der politischen Praktiken der Laufzeitverlängerung von einer Ausdehnung von Herrschafts-Eigentum ausgegangen werden, da vorherrschende zentrale Eigentumsstrukturen der Energieproduktion gestärkt werden. So kritisieren LINKE, GRÜNE und SPD dass den ,großen Vier' ein großer Teil der zusätzlichen Gewinne ${ }^{78}$ durch die Laufzeitverlängerung der Atomenergie überlassen werde (SPD 28.10.2010: 7169; LINKE 28.10.2010: 7176; GRÜNE 28.10.2010: 7178) - während die sozial-ökologischen Auswirkungen der Laufzeitverlängerung vergesellschaftet werden (vgl. Kapitel 5.2.10). Zwar soll ein Teil dieser Gewinne durch eine Kernbrennstoffsteuer abgeschöpft werden, diese sei aber nicht ausreichend, da sie zeitlich befristet sei und die Konzerne dagegen klagen könnten (SPD 28.10.2010: 7185). „Keiner von uns weiß, wie die Auseinandersetzung endet [...]. Das will ich Ihnen vorher gesagt haben, weil Sie eine so leichtfertige Politik im Interesse der Konzerne machen“ (LINKE 28.10.2010: 7176). ${ }^{79}$

Diese Entwicklung im Zusammenhang mit den politischen Praktiken der Laufzeitverlängerung fällt mit der Etablierung des Narrativs ,technokratisch-managementorientierten Ökokonsenses‘ zusammen: Denn in diesem Rahmen werden auch Artikulationsmuster, die ursprünglich zur Begründung transformativ oder reformistisch ausgerichteter politischer Praktiken herangezogen wurden, angeeignet. ${ }^{80}$ Die

77 Vgl. z.B.: „Wegen ihrer großen Sicherheitsrisiken mit der Gefahr unübersehbarer Schäden ist die Atomkraft nicht zu verantworten“ (Koalitionsvertrag 1998: 15).

78 Die LINKE spricht von Zusatzgewinnen zwischen 67 und 127 Milliarden Euro (LINKE 28.10.2010: 7176). Auch die Strompreise würden durch die Atomenergie nicht billiger, da der Preis über die Grenzkosten der teureren fossilen Kraftwerke bestimmt werde. Die Differenz seien Profite, das „sacken die Konzerne ein“ (LINKE 28.10.2010: 7196).

79 Die Brennstoffsteuer wird tatsächlich 2016 nicht verlängert und nachträglich als verfassungswidrig eingestuft. Die Gelder müssen wie hier von der Opposition befürchtet zurückbezahlt werden. Das Bundesverfassungsgericht stellt im April 2017 fest: „Da sich die Kernbrennstoffsteuer nicht dem Typus der Verbrauchsteuer im Sinne des Art. 106 GG zuordnen lässt, fehlte dem Bundesgesetzgeber die Gesetzgebungskompetenz für den Erlass des Kernbrennstoffsteuergesetzes (KernbrStG)“ (www.bundesverfassungsgericht.de). Die Bundesregierung muss 6,3 Milliarden Euro zurückzahlen (www.faz.net - Brennelementesteuer).

80 So begründet die CDU bspw. das Energiekonzept mit der Laufzeitverlängerung folgendermaßen: „Das ist eine Revolution im Prozess. Das ist eine grundlegende Umgestaltung un- 
herrschaftliche Strukturierung gesellschaftlicher Naturverhältnisse wird so weiter verdeckt, einer herrschaftsärmeren Zukunftsgestaltung wird entgegengewirkt.

\section{EEG-Novellen 2012, 2014 und 2016/2017}

Nach „Fukushima“ ist in Zusammenhang mit „Energiewende“ von einem „tiefgreifenden Umbau unserer Energieversorgung“ (Angela Merkel 09.06.2011: 12961) die Rede. Dass dieser „Umbau“ allerdings nicht auf ökonomische Strukturen abzielt, wird schnell deutlich: Die „Energiewende“ solle an den Markt angepasst werden, nicht umgekehrt (z.B. FDP 29.03.2012: 20308). Der „Umstieg“ solle nach den Leitlinien herkömmlicher Marktrationalität gestaltet werden (vgl. Kapitel 5.2.4): „Diesem Ziel dient die Novelle des Erneuerbare-Energien-Gesetzes“ (Angela Merkel 09.06.2011: 12962). Die politischen Praktiken zur „Energiewende“ der Mehrheitsgruppe können im Kontext des fantasmatischen Narrativs ,marktwirtschaftlicher Energiepolitik' interpretiert werden. Entsprechend entfalten diese politischen Praktiken in Bezug auf ökonomische Strukturen eher eine beharrende Wirkung, wie in Kapitel 5.3.2 gezeigt wurde. Gleichzeitig zeigt sich in Bezug auf das EEG nach „Fukushima“ eine von Fraser (2001: 52) beschriebene Logik affirmativer Maßnahmen: So führt das EEG als Mechanismus affirmativer Umverteilung zu einer öffentlich sichtbaren ständigen Erhöhung der EEG-Umlage, d.h. der Kosten, die auf den Strompreis umgelegt werden. Die erneuerbaren Energien (und mit ihnen die vielen kleinen, gegensätzlich zu ,den großen Vier' verorteten, Produzent*innen) erscheinen dadurch als besonders hilfsbedürftig, als Empfänger*innen einer besonderen Behandlung. So haftet ihnen das Stigma der vermeintlichen Privilegierung an in Verbindung mit dem Vorwurf, dass sie zu teuer seien. ${ }^{81}$ Die FDP spricht beispielsweise von der „gemütliche[n] Hängematte der Einspeisevergütung“, die ,abgehangen“ werden müsse (FDP 29.03.2012: 20308). ${ }^{82}$ Fraser (2001: 52) bezeichnet diese „stigmatisierende Anerkennungsdynamik“ als „praktischen Anerkennungseffekt“ affirmativer Umverteilung. Übertragen auf die „Energiewende“ impliziert dies eine soziale Abwertung erneuerbarer Energien. Politische Praktiken, deren Ziel ursprünglich „der Ausgleich von Ungerechtigkeiten in der Verteilung" war, schaffen im Endeffekt „Ungerechtigkeiten in den Anerkennungsbeziehungen“ (ebenda): Während die gesellschaftlichen Kosten der konventionellen Energie im „Energiewende“ - Diskurs kaum thematisiert werden, werden die erneuerbaren Energien unter Rückgriff auf eine komplexitätsreduzierende Story-Line, welche die Kosten in den Mittelpunkt stellt (vgl. Kapitel 5.2.5), als zu teuer dargestellt und im Rahmen des Narrativs ,marktwirtschaftliche Energiepolitik‘ zur Gefahr für eine erfolgreiche Volkswirtschaft (vgl. Ka-

seres Landes. Wir führen sie durch, weil wir glauben, dass sie dem Klimaschutz dient, und Klimaschutz ist unsere Lebensgrundlage“ (CDU 28.10.2010: 7179).

81 „Glauben Sie denn, dass die Verbraucherinnen und Verbraucher bereit wären, doppelt so viel zu bezahlen, obwohl das nicht durch die Marktpreise gerechtfertigt ist? Wir müssen die Kosten der Energiewende im Blick behalten“ (CDU 29.03.2012: 20305).

82 Interessant ist hier auch die begriffliche Analogie zum vielfach bemühten Bild der, sozialen Hängematte', die Ausdruck desselben Effektes affirmativer Umverteilungsmaßnahmen durch die Sozialversicherungssysteme ist. 
pitel 5.2.4) stilisiert. ${ }^{83}$ Den Kosten durch Zerstörung der Lebensgrundlagen wird gleichzeitig in der schreckensverheißenden Dimension des Narrativs keine Aufmerksamkeit geschenkt und Kosten durch Subventionen konventioneller Energieträger werden durch die jeweilige Mehrheitsgruppe im Parlament ausgeblendet (vgl. Kapitel 5.3.2).

Durch die einseitige Orientierung der Kostendebatte auf die EEG-Umlage als Finanzierungsinstrument erneuerbarer Energien scheint neben dem stigmatisierenden Effekt die Ausdehnung von Herrschafts-Eigentum verdeckt und begünstigt zu werden: So kritisieren vor allem LINKE und GRÜNE (vgl. z.B. GRÜNE 29.03.2012: 20286; LINKE 24.03.2011: 11308), dass der Börsenstrompreis (auch) durch die strompreissenkenden Effekte erneuerbarer Energien ${ }^{84}$ gesunken sei - stärker als die EEG-Umlage angestiegen sei. Dennoch würden die Unternehmen die EEG-Umlage als Begründung für Preiserhöhungen heranziehen. ${ }^{85}$ „Das heißt, das Ganze ist eine Gelddruckmaschine“ (LINKE 30.06.2011: 13390). Dadurch finde eine Umverteilung von Privatpersonen zu Energieversorgungsunternehmen und energieintensiver Industrie statt, die von der Umlage befreit ist. ${ }^{86}$ Preiserhöhungen, die aus Profitinteresse erfolgen, werden so den erneuerbaren Energien zugeschrieben, die stigmatisierende Anerkennungsdynamik wird durch die Profitrationalität der Marktökonomie verstärkt. Diese Dynamik in den Debatten um erneuerbare Energien schafft die Voraussetzungen dafür, das EEG - und somit das Aufbrechen der Dichotomie zentral/ dezentral - als Instrument zum Abbau von Ungerechtigkeit durch affirmative Umverteilung zu delegitimieren. Die EEG Novelle 2012 ist bereits als erste Konsequenz dieser Delegitimation zu werten, da die Grundlage zur Abschaffung der festen Ein-

83 Vgl. z.B.: „Die Debatte über die Verteilung der EEG-Umlage lenkt von dem Problem ab, dass sie inzwischen zu einer massiven Belastung geworden ist. Wir können doch nicht so tun, als ob die Steigerung der EEG-Umlage in den letzten Jahren kein Problem gewesen wäre [...]. Deswegen glaube ich nicht, dass die Industriebefreiungen das Problem sind, sondern eine in den letzten Jahren drastisch gestiegene EEG-Umlage“ (SPD 10.04.2014: 2435).

84 „Beispielsweise führt eine hohe Einspeisung von Windenergie zu einer Verringerung der nötigen Residuallast, welche durch teurere konventionelle Kraftwerke, wie Gas- oder Ö1kraftwerke gedeckt werden muss“" (Schomerus/Henkel 2012: 27). Daher haben erneuerbare Energien eine strompreissenkende Wirkung.

85 Diese Einschätzung wird durch eine Studie des Umweltbundesamtes aus dem Jahr 2011 bestätigt. Darin heißt es: „Den Anstieg der EEG-Umlage im Jahr 2011 um rund 1,5 Cent/kWh gegen- über 2010 nutzten viele Stromversorger als Argument für starke Strompreiserhöhungen. Diese Argumentation ist jedoch falsch, denn der Erhöhung der EEGUmlage stehen erhebliche Kostensenkungen an anderer Stelle gegenüber, vor allem bei den Strombeschaffungskosten. $\mathrm{Zu}$ verdanken ist dies auch dem Ausbau der erneuerbaren Energien, der zu einem Rückgang der Preise an der Strombörse führte“ (Umweltbundesamt 2011: 9).

86 „Es ist eine Umverteilung von den Kleinen zu den Großen, die hier stattfindet“ (LINKE 01.06.2016: 16986). Es könne nicht sein, „dass die privaten Verbraucher zahlen und am Ende nur die Industrie profitiert. Das hat nichts mit Kostengerechtigkeit zu tun“ (GRÜNE 10.04.2014: 2441). 
speisevergütung gelegt wird, von der vor allem Kleinproduzent*innen profitieren (Schomerus/Henkel 2012: 19; vgl. Kapitel 6.2). Auch hier wird zumindest der Status von Herrschafts-Eigentum, als Kleinproduzent*innen ausschließendes Eigentum großer Unternehmen gestärkt.

Die Novellierungen des EEG der 18. Legislaturperiode schwächen dessen Wirkungen in Richtung affirmativer Gerechtigkeit weiter ab, indem es immer mehr an marktwirtschaftliche Rationalität angepasst wird (,mehr Markt und mehr Wettbewerb" (CDU 27.06.2014: 3935)). Diese Abgabe politischer Gestaltungsmacht an den Markt wird als , vernünftig ' konstituiert. ${ }^{87}$ Gleichzeitig verstärkt sich in der 18 . Legislaturperiode die ,stigmatisierende Anerkennungsdynamik' in Bezug auf erneuerbare Energien - in Verbindung mit dem Katastrophenszenario der ,Deindustrialisierung ‘ wird immer wieder das Bild einer Bedrohung für den deutschen Wirtschaftsstandort durch die „Energiewende“ erzeugt (vgl. Kapitel 5.2.3 und 5.3.2). Es ist von einer „Last“, die man sich „aufgeladen“ (CDU 10.04.2014: 2439) habe die Rede oder von „drastische[n] Fälle[n] von Überförderung“ (SPD 27.04.2014: 3932; vgl. Kapitel 5.2.5). Ob sich Herrschafts-Eigentum durch das Ausschreibungsdesign im EEG 2016/2017 langfristig weiter ausbreitet, bleibt abzuwarten. Es deutet sich aber bereits an, dass die Teilnahme kleinerer Akteur*innen erschwert ist und sich Bürger*innenenergiegesellschaften vermehrt zu profitorientierten Unternehmen wandeln, in denen komplexe Eigentumsstrukturen privater Unternehmen die Teilhabe von Bürger*innen ersetzen (vgl. Kapitel 5.2.8).

Ein Ansatz affirmativer Umverteilung und affirmativer Anerkennung lässt sich in der 18. Legislaturperiode am ehesten bei der Einführung des Mieterstromgesetzes erkennen (vgl. Kapitel 5.2.8). Die Mieter*innen profitieren und partizipieren am Ende jedoch nur passiv von und an den erneuerbaren Energien. Zwar sinken im Rahmen eines Mieter*innenstrommodells die Strompreise für sie, die Stromproduktion bleibt aber in der Hand der Hausbesitzer*innen - Eigentumsverhältnisse bleiben unangetastet (vgl. Kapitel 5.2.8).

\section{Energetische Gebäudesanierung}

Aber nicht nur im Zuge der EEG-Novellen wird nach „Fukushima“ die „Energiewende“ für die Stärkung von herrschaftlichen Eigentumsstrukturen genutzt. Durch die einseitige Fokussierung des Diskurses auf den Bereich der Marktökonomie werden Gerechtigkeitsfragen zunehmend in das ,Außen“ der „Energiewende“ gedrängt. So wird 2013 eine Änderung im Mietrecht beschlossen, die der „Energiewende“ im Wärmebereich dienen soll, indem die Hürden für eine energetische Sanierung gesenkt werden (Koalitionsvertrag 2009: 28; vgl. auch Kapitel 5.1.2). Dabei werden die Kosten von Sanierungen einseitig auf die Mieter*innen umgelegt. ${ }^{88}$ Darüber hinaus

87 Vgl. z.B.: „Heute ist für mich eine Premiere: Ich diskutiere über das EEG, ohne dass mir dabei gleich das Messer in der Tasche aufgeht [...]. Genau genommen habe nicht ich mich verändert, sondern das EEG hat sich verändert, und zwar in einer vernünftigen Weise $[\ldots]^{\text {“ }}$ (CDU 27.06.2014: 3935).

88 Dies wird von der Opposition kritisiert: Vgl. z.B.: „Unter dem Vorwand der notwendigen energetischen Sanierung verschieben Sie mit dem vorliegenden Entwurf eines Mietrechtsänderungsgesetzes alle Lasten auf die Schultern der Mieterinnen und Mieter [...]“ 
werden sogar zusätzliche langfristige Profite für die Vermieter*innen generiert, da die Mieterhöhung von 11\% der Sanierungskosten pro Jahr ( 5559 BGB 2013) auch über die vollständige Abbezahlung der Sanierungskosten hinaus Bestand haben darf. ${ }^{89}$ Dass energiepolitische Praktiken, die sich im Kontext des Narrativs des ,technokratisch-managementorientierten Ökokonsenses` zeigen, eine Festigung bestehender Externalisierungsstrukturen bewirken können, lässt sich hier beispielhaft aufzeigen. „Wir wollen die Eigentümer ermutigen, ihre Miethäuser und Mietwohnungen energetisch auf den aktuellen Stand zu bringen. Damit leisten wir in der Rechtspolitik unseren Beitrag zur Energiewende“" (FDP 13.12.2012: 26286). Während der Fokus auf dem als aktiv konstituierten, dem Ökonomischen zugewiesenen Eigentümer liegt, erscheinen die Mieter*innen im Gegensatz zum aktiven Eigentümer-Investor eher als passiv. Die Änderung des Mietrechtes soll bewirken, dass auch sie ihren Beitrag zur „Energiewende“ leisten - ohne, dass sie an Entscheidungen über Sanierungen beteiligt werden. ${ }^{90}$ Der Grundsatz von Eigentum, das verpflichtet, wird dabei ausgeblendet und mit dem Eigentum an Wohnraum zusammenhängende Machtverhältnisse werden nicht artikuliert - so erscheint die Finanzierung durch die Mieter*innen sogar als ,gerecht":

„Die Reform des Mietrechtes ist ein wichtiger Baustein der Energiewende [...]. Deswegen brauchen wir Rahmenbedingungen, die Eigentümer nicht von Investitionen abhalten [...]. Natürlich stellt das eine Belastung der Mieter dar. Aber wir können unsere ehrgeizigen Klimaschutzziele nur erreichen, wenn alle an einem Strang ziehen. Das ist eine gesamtgesellschaftliche Aufgabe, deren Lasten gerecht verteilt werden müssen. Daran müssen alle mitwirken. Deswegen ist es, so glaube ich, gerechtfertigt, dass auch Mieter hier ihren Beitrag zum Gelingen der Energiewende leisten“ (CDU 13.12.2012: 26290f.).

Politische Praktiken affirmativer Umverteilung werden hier in ihr Gegenteil verkehrt: So ziele die Gesetzesreform explizit darauf die Rechte der Vermieter*innen zu Lasten der Mieter*innen zu stärken, denn durch vergangene Reformen seien Mieterrechte ,immer weiter aufgebaut“ worden, es sei „eine Schieflage“ entstanden, die nun

(LINKE 13.12.2012: 26291f.). Vgl. z.B.: „Sie versuchen unter dem Vorwand der Energiewende, Mieterinnen- und Mieterrechte abzubauen“ (GRÜNE 13.12.2012: 26293).

89 Diese Änderung im Mietrecht erfolgte 2013 mit dem §559 BGB und ermöglichte umfassende Mietsteigerungen. Laut §559 BGB (2013) kann der Vermieter 11\% p.a. der Sanierungskosten für die Wohneinheit auf den Mieter durch Mieterhöhung umlegen. Folglich sind nach ca. 9 Jahren die Sanierungskosten durch Mieterhöhung vollständig gedeckt. Da die Mieterhöhung aber dauerhaft gilt, stellen die 11\% p.a. der Sanierungskosten ab dann für den*die Vermieter*in einen Reingewinn dar. Seit Januar 2019 wurde diese Umlage auf $8 \%$ reduziert. Zudem darf sich die Miete nun innerhalb von 6 Jahren nicht mehr um mehr als 3 Euro je Quadratmeter Wohnfläche erhöhen ( $\$ 559$ BGB 2019).

90 Vgl. z.B.: „Ihnen [den Mieter*innen] erlegen wir auf, diese Sanierungsmaßnahmen zu dulden [...] und für die Dauer von drei Monaten die Miete nicht zu mindern; denn [...] wenn in einem Mehrfamilienhaus 5, 10 oder 20 Mieter die Miete mindern, dann ist das schon ein gewaltiges Hindernis für den Eigentümer bei der Durchführung dieser Modernisierung“ (FDP 13.12.2012: 2686f.). 
„wieder ins Lot zu bringen“ sei, indem die „Anreize für Investitionen“ erhöht werden (FDP 13.12.2012: 2686). ${ }^{91}$

\section{Bewertung}

Insgesamt materialisieren sich transformative Ansätze, die bei der Bedeutungsgenerierung von „Energiewende" abgesehen vom marginalisierten Narrativ des ,sozialökologischen Umbaus“ überwiegend im Kontext des Narrativs, ökologische Modernisierung' artikuliert werden, nur unzureichend in Form politischer Praktiken. Vor dem Hintergrund der dieser Arbeit zu Grunde liegenden sozial-ökologischen Forschungsperspektive konnten hierarchisch strukturierte gesellschaftliche Naturverhältnisse sowie Verteilungs- und Anerkennungsverhältnisse, die auch unter rot-grün nur wenig adressiert werden, sichtbar gemacht werden. Analog zur nachgezeichneten Wirkrichtung des ,technokratisch-managementorientierten Ökokonsenses ' (vgl. Kapitel 5.4), ist die Wirkung politischer Praktiken im Rahmen von „Energiewende“ nach „Fukushima“ auch mit dem Ansatz von ,Affirmation' und ,Transformation“ nach Fraser (2001) eher als beharrend ${ }^{92}$ einzustufen. Vor dem Hintergrund einer dominierenden Marktrationalität etablieren sich somit keine politischen Praktiken, die auf den Abbau von Herrschaft abzielen. Solche Effekte, die sich mit dem EEG in der Perspektive von Transformation zeigten, werden nach „Fukushima“ eher zurückgedrängt. Eine herrschaftsärmere Zukunftsgestaltung ist insgesamt kaum als Objekt politischer Praktiken im Rahmen von „Energiewende“ nach „Fukushima“ erkennbar.

Aspekte transformativer Umverteilung und Anerkennung vor dem Hintergrund der dieser Arbeit zugrundeliegenden Perspektive werden ab der 17. Legislaturperiode überwiegend von GRÜNEN und LINKEN artikuliert. Bereits ab der 14. Legislaturperiode artikuliert die PDS und später die LINKE Forderungen im Kontext des Narrativs ,sozial-ökologischer Umbau', wobei Potenziale für transformative Umverteilung und Anerkennung sichtbar werden. In diesem Kontext werden die zugrunde liegenden Produktions- (bzw. das „Wachstumsmodell“) (PDS 25.02.2000: 8436), Verteilungs- (z.B. LINKE 29.03.2012: 20277) und Teilhabeverhältnisse (vgl. die Forderung nach Demokratisierung, z.B. LINKE 24.03.2011: 11284) als Hindernis für die Bewältigung sozialer und ökologischer Krisen adressiert. Insgesamt bringen sich aber PDS und LINKE (mit Ausnahme der 16. Legislaturperiode) quantitativ betrachtet relativ wenig in den Diskurs ein (vgl. Abbildung 7). Dies kann man als Hinweis deuten, dass die herrschaftliche Strukturierung gesellschaftlicher Naturverhältnisse und deren Verwobenheit mit Gesellschaftsverhältnissen kein Kernthema der LINKEN, bzw. möglicherweise innerhalb der LINKEN marginalisiert ist. Das ist aber kein Gegenstand der vorliegenden Untersuchung.

91 Der Berliner Mieterverein e.V. kommt in einer empirischen Kurzstudie über 200 Maßnahmen im Berliner Mietwohngebäudebestand 2017 zu dem Schluss, dass über das Gesetz abgedeckte Modernisierungsmaßnahmen zu einem ,durchschnittliche[n] Mietanstieg um 2,44 $€ / q m$ bzw. 186,37 € absolut im Monat“ führen, was ,gemessen an der durchschnittlichen ortsüblichen Vergleichsmiete im Mietspiegel 2015 einen Anstieg von fast $42 \%$ “ bedeutet. Die Senkung der Heizkosten stehe dazu in keinem Verhältnis (Wild 2017: 15).

92 Beharrung ist hier nicht dasselbe wie Affirmation, da Praktiken affirmativer Umverteilung und Anerkennung nach obiger Einordnung eher als reformistisch einzustufen wären. 
